INTERNATIONAL JOURNAL OF ORGANIC EVOLUTION

PUBLISHED BY

THE SOCIETY FOR THE STUDY OF EVOLUTION

Vol. 48

December 1994

No. 6

Evolution, 48(6), 1994, pp. 1747-1763

\title{
MECHANISMS OF LARGE-SCALE EVOLUTIONARY TRENDS
}

\author{
DaNiEl W. MCShea \\ Museum of Paleontology, University of Michigan, Ann Arbor, Michigan 48109
}

\begin{abstract}
Large-scale evolutionary trends may result from driving forces or from passive diffusion in bounded spaces. Such trends are persistent directional changes in higher taxa spanning significant periods of geological time; examples include the frequently cited long-term trends in size, complexity, and fitness in life as a whole, as well as trends in lesser supraspecific taxa and trends in space. In a driven trend, the distribution mean increases on account of a force (which may manifest itself as a bias in the direction of change) that acts on lineages throughout the space in which diversification occurs. In a passive system, no pervasive force or bias exists, but the mean increases because change in one direction is blocked by a boundary, or other inhomogeneity, in some limited region of the space. Two tests have been used to distinguish these trend mechanisms: (1) the test based on the behavior of the minimum; and (2) the ancestor-descendant test, based on comparisons in a random sample of ancestor-descendant pairs that lie far from any possible lower bound. For skewed distributions, a third test is introduced here: (3) the subclade test, based on the mean skewness of a sample of subclades drawn from the tail of a terminal distribution. With certain restrictions, a system is driven if the minimum increases, if increases significantly outnumber decreases among ancestor-descendant pairs, and if the mean skew of subclades is significantly positive. A passive mechanism is more difficult to demonstrate but is the more likely mechanism if decreases outnumber increases and if the mean skew of subclades is negative. Unlike the other tests, the subclade test requires no detailed phylogeny or paleontological time series, but only terminal (e.g., modern) distributions. Monte Carlo simulations of the diversification of a clade are used to show how the subclade test works. In the empirical cases examined, the three tests gave concordant results, suggesting first, that they work, and second, that the passive and driven mechanisms may correspond to natural categories of causes of large-scale trends.
\end{abstract}

Key words. - Diffusion, driven trend, evolutionary trends, passive trend, trend mechanisms.

Received September 17, 1993. Accepted February 22, 1994.

Large-scale evolutionary trends may be passive or driven. Figure 1 shows two trends that were generated by a computer model for simulating the diversification of a clade: figure $1 \mathrm{~A}$ is passive and figure $1 B$ is driven. In both, the clade begins as a single lineage, a single species, at some small value of an unspecified dimension (horizontal axis), and as time (vertical axis) passes and diversification proceeds, the mean value for the clade as a whole increases. These changes are trends in that the direction of change in the mean

Present address: Santa Fe Institute, 1399 Hyde Park Road, Santa Fe, New Mexico 87501. is persistent, if not monotonic. They are large scale in that they occur in a large group of species, a clade, rather than a single lineage, and they occur over a relatively long span of time, enough time that they incorporate a number of speciation events.

The two trends were produced by the same computer program but using very different parameters. In the passive system (fig. 1A), speciation and anagenetic change were both unbiased with respect to the horizontal dimension. That is, decreases and increases were equally likely. However, decreases that would have moved existing lineages (or created new ones) to the left 

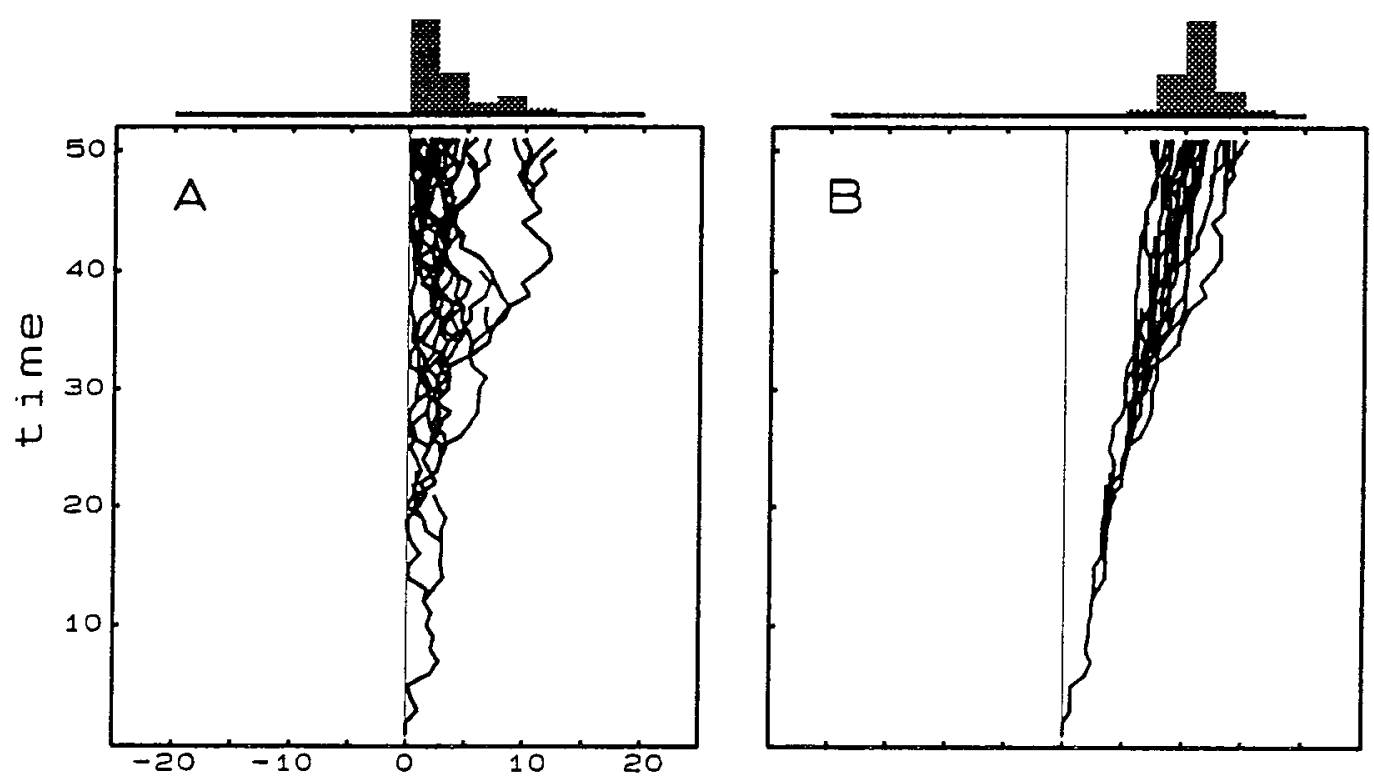

Fig. 1. Simulation of the diversification of a clade in a passive (A) and a driven (B) system. The horizontal dimension is unspecified, but it could be almost any variable (e.g., size, metabolic rate, speciation rate, location in space). See text for discussion of the general features of the computer model. In the passive system, a cushioning boundary is present at zero, meaning lineages that would otherwise cross the boundary are assigned their original value. In the driven system, no boundary is present, but increases are more likely than decreases. Histograms above show distributions for the clades after 50 time units.

of the vertical axis were forbidden. In other words, the vertical axis was an impenetrable lower bound, and the trend was the result of passive diffusion away from the bound. In the driven process (fig. 1B), no bound existed, but instead there was a bias, such that when either branching or anagenetic change occurred, it was usually to the right.

Stanley (1973) introduced the notion that evolutionary trends may be passive in the sense described. He argued that mean size is expected to increase if groups originate at small size, near a lower bound, and that this mechanism accounts for Cope's rule, the well-known tendency for groups to increase in size in evolution (reviewed in LaBarbera 1986; see also Damuth 1993). The presence of the lower bound, he noted, partly accounts for the tendency of size distributions to be right or positively skewed (as in fig. 1A). He gave the lower bound a physical interpretation, suggesting that it might result from physiological constraints or other design limitations. Stanley's model also incorporated a selective component in that groups were said to originate below, and then move toward, their size optima.

Fisher (1986) presented the passive mecha- nism in more general and purer form, describing it as "diffusion within a structured design space." He argued that if an ancestral morphology is located asymmetrically relative to a set of constraints, then even if change is unbiased (i.e., if no movement toward an optimum occurs), the longer and more conspicuous trends in descendant lineages will be away from the more proximal constraint. It follows from Fisher's argument that the mean will also move away from the more proximal constraint. Gould (1988) described this process as an increase in variance in the presence of a lower bound.

In the driven system, the bias might be caused by selection: when new species arise, or when anagenetic change occurs, increases occur more often than decreases on account of the advantages to individuals (or species) of higher values of the dimension in question. If the dimension is size, a bias of this sort might account for Cope's rule (Newell 1949; Bonner 1988; McKinney 1990b). Note that a system may be driven in other senses. In principle, for example, a driven system could be one in which increases and decreases are equally likely, but the magnitude of increases is larger, although such a mechanism 
has not, to my knowledge, been proposed for any real large-scale trend.

The same two mechanisms have been invoked to explain other large-scale trends. For example, Bonner (1988) has suggested that increases in complexity may be favored by natural selection, and Saunders and Ho (1976) have suggested that complexity increases may occur more frequently than decreases for structural or developmental reasons. Salthe (1993), Wicken (1987), Brooks and Wiley (1988), and others have argued that increases in complexity are driven eutrophically. In all of these mechanisms, the suggestion is that increases are more likely; that is, that a bias is present in the evolutionary process. In present terms, the increase is said to be driven. However, Maynard Smith (1970) proposed that complexity increases, at least those early in the history of life, were passive. He argued that if the first organisms were and had to be as simple as possible (i.e., if life originated at or near a complexity lower bound), then subsequent evolution could have been only in the direction of greater complexity. This proposal could be easily extended to explain the overall pattern of complexity increase in the history of life, assuming such an increase has in fact occurred (McShea 1991).

A driven mechanism has been proposed to explain large-scale increases in absolute fitness (e.g., Van Valen 1973; Vermeij 1987). And Vermeij (1987), in considering objections to his escalation theory, raised the possibility that the increase in fitness may have occurred passively.

In addition to changes in morphology, population movements in ecological time may be passive or driven. A population shift is driven if it is the result of active migration, perhaps from a less favorable to a more favorable habitat, and passive if it results from bounded diffusion, such as the dispersal of a population in a favorable habitat that is bounded by an unfavorable one (Skellam 1951; Toft and Mangel 1991). Likewise, higher taxon movements in geological time may be passive or driven. Bottjer and Jablonski (1988; see also Jablonski and Bottjer 1990; Sepkoski 1991) described two spatial patterns for taxa originating in onshore (nearshore or innershelf) environments: "expansion," which involves migration offshore while leaving representatives onshore; and "retreat," or migration offshore leaving no onshore species. In principle, expansion could be a passive process (Jablonski and Bottjer 1990) analogous to the passive pro-

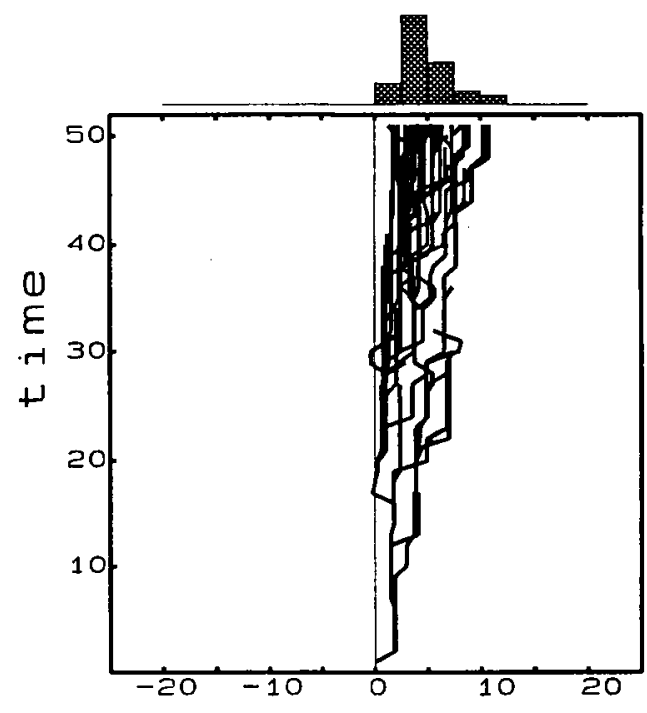

FIG. 2. A driven system, again with a branching bias and no boundary (as in fig. 1B), but in this case with a low probability of change in each time interval. The resulting distribution is skewed, and its minimum stays close to the starting value (zero), effectively mimicking a passive system (fig. 1A).

cess in figure 1A (although such a pattern could also be driven; see figure 2 and discussion below), and retreat could be a driven process analogous to that in figure $1 \mathrm{~B}$.

As these examples suggest, the range of application of the passive-driven distinction is quite broad. The dimension along which change occurs, what McKinney (1990a) called the "state variable," may be morphological (e.g., size, complexity), cladogenetic (e.g., speciation rate, extinction rate), or ecological (e.g., fitness, geographic range) (Raup 1988). Fisher (1986) and Raup (1988) listed a number of other possibilities.

Figures 1A and 1B suggest various methods for distinguishing driven and passive systems. First, a test based on the behavior of the minimum: in a passive process, the distribution minimum ought to move to the boundary and stay near it (Gould 1988). In a driven process, however, the minimum is expected to increase significantly, at least in the long run. Jablonski (1987) used the minimum test to distinguish passive from driven increases in size in Cretaceous bivalves and gastropods. Boyajian and Lutz (1992) used this test to investigate the mechanism of change for a trend in the complexity of ammonoid sutures. 

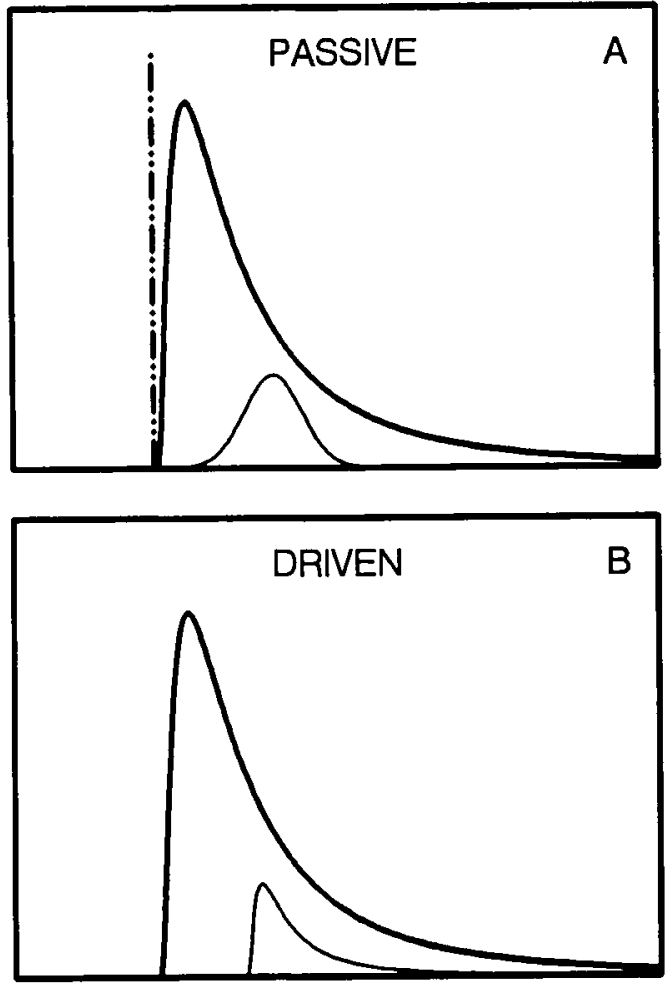

Fig. 3. The subclade test. In a passive system (A), the parent clade is skewed by the boundary, but a subclade drawn from the right tail of the parent clade is not. In a driven system (B), both the parent clade and the subclade experience the same forces, and thus both are skewed. Subclades are true monophyletic groups not random samples of lineages (see text).

Second, if portions of the phylogeny are known, a bias in the direction of change may be detectable in a random sample of ancestor-descendant comparisons. If the system is passive, increases and decreases in the state variable should occur about equally often (Fisher 1986; McShea 1993). MacFadden (1986), McShea (1993), and Boyajian (pers. comm. 1993) used this test to examine the mechanism for trends in horse body size, vertebral-column complexity, and ammonoidsuture complexity, respectively.

Third, the passive process tends to produce skewed distributions (fig. 1A, and noted in Gould 1988; Stanley 1973), which raises the possibility that skewness might provide a basis for distinguishing the two kinds of system. Unfortunately, a test based solely on clade skewness would be unreliable. Simulations (see below) show that, under certain conditions, driven processes may produce skewed distributions, thereby mimicking passive processes, at least in the short run.
Figure 2 shows a driven system in which the mean increases, and the distribution becomes skewed, but the minimum stays very close to the starting value.

Here, a new test is developed in an attempt to improve our ability to distinguish driven and passive trends. The principle behind the new test is this: in a trend in which a clade's distribution becomes skewed, the skewness of a subclade drawn from the tail of that distribution will tend to reflect a local regime of constraints or selective forces or both, whereas the clade as a whole will reflect a global regime. Specifically, if the clade's distribution is skewed by the presence of a lower bound, then a subclade drawn from the tail of that distribution, far from that bound, will have no tendency to be skewed (fig. 3A). However, if the clade is skewed as a result of a branching bias that operates uniformly across the space (that is, uniformly over the range of the dimension in question), then subclades from the tail of the distribution will likewise be skewed (fig. 3B).

"Subclade" is understood here as a monophyletic subset of a temporal cross-section of a larger parent clade. (The larger clade is "parental" only in that it is the clade from which the subclade is drawn; it is not ancestral.) Both the parent clade and its subclade have distributions in the dimension of interest; in the protocol adopted here, a subclade drawn from the tail is defined as a monophyletic subset chosen such that the mean of its distribution is greater than the mean of the parent distribution.

The subclade test has the virtue that it requires only two distributions, an early or ancestral distribution and a skewed terminal or descendant distribution, and no information about the intervening pattern of branching. Actually, the subclade test requires an ancestral distribution only to establish that a trend has occurred. Thus, for trends in which ancestral values can be assumed to be low, but the ancestral distribution is unknown and may not be knowable (a possible example is metabolic rate in vertebrates), the trend mechanism can still be investigated using only a modern distribution. However, the test does have certain limitations, and in some cases it depends on assumptions that may be sufficiently unrealistic as to render it useless. These are discussed later.

The logic of these tests works for decreasing trends as well as increasing. Evidence for a driven decrease would be a decrease in the maximum, a significantly greater number of decreases in a random sample of ancestor-descendant compar- 
isons, and a significantly negative mean skew for subclades drawn from the tail of a left (negatively) skewed parent distribution. For simplicity, a trend to the right and a right (positive) skew are assumed in the discussion that follows.

Most large-scale trends are probably quite complex, in the sense that they result from a variety of different causes operating at different times and over a variety of different taxonomic, spatial, and temporal scales. Given this complexity, the prior expectation was that most trends would not be readily classifiable as either purely driven or purely passive, but rather that most would lie somewhere in between or share features of both. The various tests capture different aspects of trend dynamics; thus, the further expectation was that in empirical cases test results would not agree. It was surprising, therefore, that in the cases analyzed, all test results did agree, raising the possibility that the passive-driven distinction in fact corresponds to a natural division.

\section{Passive and Driven Systems}

The distinction between the passive and driven mechanisms is not necessarily that between selection and developmental constraints, nor even between internal and external factors. A driven system such as the one shown in figure $1 B$ is a fairly standard model for a selection-driven system, whether selection occurs among species, populations, or individuals. But note that the passive system in figure $1 \mathrm{~A}$ could also be a consequence of selection, perhaps if the boundary were a result of selection acting only against very small species (or populations of small individuals). Alternatively, the branching bias of the system in figure 1B could, in principle, be the result of directed speciation (Stanley 1979), developmental channeling (Alberch 1980), or even internal drives of various kinds (Cope 1871). Likewise, a boundary could be the result of a developmental constraint. In principle, both boundaries and biases can result from either internal or external factors, or both.

The actual distinction being made is more general and can be understood in a number of different ways. For example, the passive spread of a growing clade away from a boundary is a kind of diffusion (Fisher 1986; McKinney 1990a), analogous to the diffusion caused by Brownian movement of particles in a suitable medium away from a source located at a boundary. Likewise, a directional bias in all or most lineages in a clade is analogous to an external force field-perhaps a gravitational or electrical field-acting on the particle-diffusion regime. The field adds a bias in the direction of particle movement, producing what Berg (1993) called "diffusion with drift." Indeed, the relation may be deeper than that of analogy: natural selection, for example, is a kind of force (Sober 1984), and selection acting on lineages throughout a morphospace is a kind of force field (see below).

Use of the word "passive" may suggest to some that the trend in a passive system is a purely chance phenomenon. As should be clear from the diffusion analogy, however, a trend in a passive system is no less inevitable than diffusion. The word passive is used mainly to emphasize that the trend occurs without the application of a pervasive forcing field. The diffusion analogy should also make clear that, at the small scale (within lineages), change should not be conceived as random or nondeterministic. Just as the movement of each individual particle in a purely diffusive system is completely determined (by its collisions with neighbors), so the morphological changes in each lineage may be determined (perhaps by selection). Diffusion is a large-scale description of the behavior of an ensemble of particles or lineages, and a passive trend is to be understood as a statistical consequence of diverse deterministic causes acting in concert at a boundary but acting unconcertedly away from the boundary.

One limitation of the analogy is that in diffusion, the particles are ordinarily understood to move independently, whereas in an evolutionary system, lineages may be constrained by ancestry.

Alternatively, the two mechanisms could be understood to refer to a difference in degree of state-space homogeneity. Figure 4 (a modification of fig. 1) represents this notion graphically. In figure $4 \mathrm{~A}$, forces (vectors) act in concert on the left side of the space, creating a boundary that resists penetration by evolving lineages. On the right side, forces act unconcertedly and produce no net effect except diffusion of the lineages away from the boundary. The system is thus heterogeneous in that the regime of forces changes at the boundary. In figure 4B, forces act more or less concertedly throughout the space, pushing lineages to the right, on average. Vector magnitudes vary in space and time, and some even point left, but this variability is distributed randomly; thus, the space is effectively homogeneous.

In this view of the passive-driven distinction, the three tests are understood as homogeneity or heterogeneity detectors. Importantly, however, 

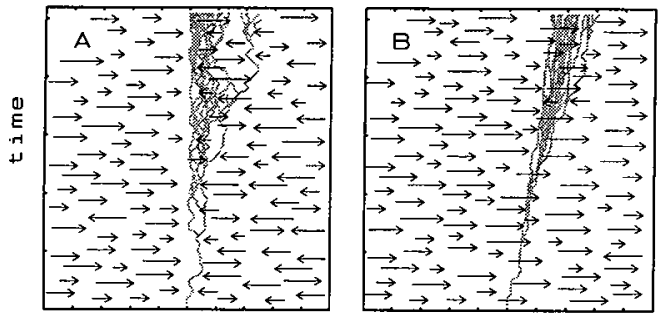

Fig. 4. Modification of figure 1 to show the sense in which a passive trend $(A)$ occurs in a heterogeneous space and a driven trend (B) in a homogeneous space, with vectors representing regimes of evolutionary forces acting in concert (force fields). In the driven system, the field is more or less homogeneous in space and time, driving the lineages to the right. In the passive system, the concerted forces are concentrated on the left, creating a barrier; on the right, the orientation of vectors is random so that no net force acts, and diffusion occurs. See text for further discussion.

because boundaries and biases may result from either selection or constraints (or both), the tests do not distinguish among the various possible causes of homogeneity or heterogeneity.

Figure 4 is somewhat misleading in two ways. First, it implies that all lineages entering a given region of the space at a given time would experience precisely the same forces, which is not the case. Every lineage experiences unique forces, and the vectors are best thought of as local averages. Second, the use of vectors suggests that the forces are external and act upon the lineages, but this need not be the case. The passive and driven mechanisms could equally well result from biases or constraints that are internal to the lineages.

Note that homogeneity and heterogeneity are scale-relative properties (Fisher 1986). At the human scale a grass lawn may be fairly homogeneous and free of boundaries, for ordinary purposes (such as mowing), whereas at an insect scale, individual blades of grass may present significant barriers, creating a fairly heterogeneous space. At a smaller scale yet, a bacterium may perceive a homogeneous environment if, for example, it never encounters (or never leaves) a blade. Similarly, any homogeneous or heterogeneous space may be considered to be so only at a certain scale.

The subclade test is therefore also scale relative. The skewness of a subclade from the tail of a skewed parent distribution should reflect the local constraint or force regime at the scale of the subclade chosen. Note that there is no expectation that a result obtained for one parent clade in one part of a space will be obtained in other regions. Just as subclades report the local regime at some small scale, so the parent clade reports the regime that is local at a larger scale.

Furthermore, homogeneity and heterogeneity are continuous variables so that, even at a given scale, real spaces need not be completely one or the other. And the degree of homogeneity may itself vary across the space. Thus, there is no expectation that, for a given parent clade, the subclade test will yield results that are consistent with other tests, even when substantial data are available.

Finally, I have referred to the passive and driven explanations as mechanisms, and it will continue to be convenient to do so, but they are more properly understood as two broad and overlapping classes of mechanism. The passive class corresponds to those mechanisms in which the distribution of forces and/or constraints is heterogeneous and the driven class to those in which the distribution is homogeneous. For example, passive trends include those resulting from a morphological boundary, as in figure $1 \mathrm{~A}$, but also those caused by a change in speciation rate or extinction rate, or even a change in the probability of change, in some region of the space in which diversification occurs. Thus, the systems represented in figures $1 \mathrm{~A}$ and $\mathrm{B}$ are nothing more than classic cases, exemplifying only a fraction of a wide range of smaller-scale mechanisms encompassed by the terms passive and driven. Finally, note that some trends do not fall in either class, such as those that occur by chance alone. (For further discussion, see Fisher 1986; Gould 1990; McKinney 1990a.)

\section{A MODEL}

The model introduced earlier is allied with a well-known class of Monte Carlo models developed for studying evolutionary processes (e.g., Raup 1977; Raup and Gould 1974; cf. Slatkin 1981). Here it is used to examine the behavior of the minimum and skewness in diversifying systems.

The model has five steps. (1) A clade begins as a single lineage at a value of zero in a onedimensional space. (2) In the first iteration (the first time unit), the lineage changes anagenetically by a quantity that is added or subtracted, with the magnitude of the change equal to the absolute value of a random variable chosen from a normal distribution with mean 0 and standard deviation $s a$ (for present purposes, $s a=1$ ). The direction of the change is determined by the pa- 
TABle 1. Protocol for the model.

\begin{tabular}{|c|c|c|}
\hline Steps & Parameter & Comment \\
\hline 1. Starting value & 0 & \\
\hline \multicolumn{3}{|l|}{ 2. Anagenesis } \\
\hline Probability of increase & pia & For an unbiased system, pia $=p d a=0.5$; \\
\hline Probability of decrease & $p d a$ & for a biased system, pia/pda $=9$ \\
\hline $\begin{array}{l}\text { Probability of change } \\
\qquad(p c a=p i a+p d a)\end{array}$ & pca & $\begin{array}{l}\text { For systems with low probability of } \\
\text { change, } p c a \ll 1\end{array}$ \\
\hline $\begin{array}{l}\text { Step size (random variable, normally distrib- } \\
\text { uted, with a mean of } 0 \text { and standard devia- } \\
\text { tion sa) }\end{array}$ & sa & $\begin{array}{l}\text { For an anagenetic system, } s a=1 ; \text { for a } \\
\quad \text { cladogenetic system, } s a=0\end{array}$ \\
\hline \multicolumn{3}{|l|}{ 3. Cladogenesis } \\
\hline Probability of branching & $p b$ & $p b=0.05$ \\
\hline $\begin{array}{l}\text { Probability of increase (when branching oc- } \\
\text { curs) }\end{array}$ & $p i$ & $p i=p i a$ \\
\hline $\begin{array}{l}\text { Probability of decrease (when branching oc- } \\
\text { curs) }\end{array}$ & pd & $p d=p d a$ \\
\hline $\begin{array}{l}\text { Probability of change (when branching occurs; } \\
\qquad p c=p i+p d \text { ) }\end{array}$ & $p c$ & $p c=p c a$ \\
\hline $\begin{array}{l}\text { Step size (random variable, normally distrib- } \\
\text { uted, with a mean of } 0 \text { and standard deviation } \\
s b \text { ) }\end{array}$ & $s b$ & $s b=1$ \\
\hline \multicolumn{3}{|l|}{ 4. Extinction } \\
\hline Probability of extinction & pe & $p e=0.05$ \\
\hline 5. Number of iterations (time) & $m$ & $\begin{array}{l}\text { When time is held constant, } m=100 \text {; } \\
\text { otherwise, } m=50,100,150,200 \text {, and } \\
250\end{array}$ \\
\hline
\end{tabular}

rameters pia, probability of anagenetic increase, and $p d a$, probability of anagenetic decrease. In an unbiased system, pia $=p d a$. Importantly, the probability of change $(p c a=p i a+p d a)$ need not equal 1 ; if $p c a$ is less than 1 , then anagenetic stasis occurs with probability $1-p c a$.

(3) The lineage is then given an opportunity to branch, which it does with probability $p b$. If branching occurs, the magnitude of the branch length (the first step taken by the new lineage) is the absolute value of a random variable chosen from a normal distribution with mean zero and standard deviation $s b$ (here, $s b=1$, so that step sizes for anagenesis and branching have the same distribution). The probability of increase if branching occurs is $p i$, which for present purposes is set equal to pia, and the probability of decrease is $p d$, which is set equal to $p d a$. (4) A boundary is established at some value $b$ (here, $b$ $=0$ for a bounded, passive system; $b=-\infty$ for an unbounded, driven system). The protocol for the behavior of lineages encountering the boundary is discussed in the next section. (5) Finally, whether branching has occurred or not, the original lineage has an opportunity to become extinct, which it does with probability pe. Subsequent time units are the same as the first, with each extant lineage having an opportunity to change anagenetically, to branch, and then to become extinct. The system evolves for $m$ time units. Table 1 summarizes the steps and variables in the model.

A run of the model is considered successful if the clade has 25 or more extant lineages at the end of $m$ time units. Using this cutoff is essential, because smaller clades tend to have few or no moderate-size subclades. This cutoff may bias the results, but in the analysis of actual cases, some minimum clade size close to 25 would have to be established for the same reason; thus, real clades would be biased in the same way and to about the same degree.

When time is a variable, $m$ is set equal to 50 , $100,150,200$, and 250 . When time is a constant, $m=100$. For most experiments with the model, extinction probability (pe) is set equal to branching probability $(p b)$, with both equal to 0.05 . If understood as a per-200,000-year rate, this rate is approximately equal to 0.25 originations or extinctions per lineage per million years, or close to the mean value that has been estimated for marine taxa in the Phanerozoic (Raup 1991). An $m$ value of 250 , therefore, corresponds to about 50 million yr of evolution. In any case, results (not shown) for simulations using a range of values from $p b=p e=0.02$ to 0.08 did not differ qualitatively from those for 0.05 .

For each successful run, the maximum, mean, 
minimum, and skew are computed. Then a search is conducted for a single subclade meeting certain requirements. Here, a subclade is considered drawn from the tail of the parent clade's distribution if the subclade's mean is greater than the parent clade's. A subclade is considered too small if it has fewer than five lineages and too large if it has more than one-quarter as many lineages as the large clade. A run is discarded if no subclade meeting these requirements can be found. Again, any bias this procedure introduces into the results would also be present in real cases. For each set of parameters, the model continues until 200 runs meeting these criteria have accumulated.

For investigating trends in morphology, it might seem that a multiplicative model (Maurer et al. 1992) would be more appropriate than the additive model described above. Size changes, for example, in both ontogeny and phylogeny are more likely to be multiplicative or proportional than additive. However, to linearize proportional changes, the conventional procedure is to take the log of all body sizes and to conduct the analysis in log space; the effect of this linearization is to render proportional changes additive. Thus, if the space in which a clade is diversifying is simply conceived as a log space, an additive model is perfectly appropriate and, indeed, formally identical to a multiplicative model operating in linear space. Actually, the additive model is more general, because it can be used both in cases in which the dimension of interest really is additive, perhaps for complexity (understood as a count of number of different parts), and also-with a simple conceptual shift to log spacefor variables that change proportionally like size.

Two configurations of the model were investigated, one to simulate a diversifying anagenetic system (i.e., a system in which change occurs both during and between branching events) and another to simulate a purely cladogenetic system (i.e., punctuated equilibrium, in which change occurs only during branching events). Parameters for modeling the anagenetic system were described above. The cladogenetic system is the same except that $s a$ is set equal to 0 . (The convention is to use the word "anagenesis" to refer to change in single lineages; note that here the phrase "anagenetic system" is shorthand for a multilineage branching system in which anagenesis occurs.) In all configurations, the assumption is that the parameters remain constant, that is, that the system is time homogeneous (Raup 1985).

\section{Passive Systems}

In a passive system, a clade encounters a boundary in an otherwise homogeneous space. In the model, the boundary was placed at zero, the starting value for the clade, and anagenetic or cladogenetic events that would otherwise have taken a lineage to some value less than 0 , were forbidden. The boundaries are understood to correspond to selective or constraint regimes that engage abruptly, at some precise value.

The effects of three types of boundary were examined: cushioning, sticky (adsorbing), and reflecting. With a cushioning boundary, a change that would otherwise have carried a lineage across the boundary is simply nullified and the lineage is instead assigned the value it had before the change occurred. With a sticky boundary, a lineage attempting to cross the boundary is assigned a value of zero, the value of the boundary itself. And with a reflecting boundary, the lineage is assigned a positive value equal to the distance it would otherwise have traveled into the negative region beyond the boundary. Cushioning or sticky boundaries seem biologically realistic; on account of some selection or constraint regime that engages at the boundary, an evolving lineage decreases less than it would otherwise. It is difficult to imagine a realistic scenario corresponding to a reflecting boundary, but it was investigated anyway to cover all the geometric possibilities. (An absorbing boundary is equivalent to a sharp extinction gradient; see below.)

All three boundaries gave very similar results; thus, only one set of results (sticky boundary) is shown (fig. 5). The mean increased (fig. 5A), and even the minimum increased, but not very much. The mean skew for the parent clade was positive (fig. 5C), but for subclades drawn from the righthand tail it was close to zero. A cladogenetic model (fig. 5B,D) gave similar although not so dramatic results. With change occurring only at branching events, the parent clade had little opportunity to spread very far from the boundary, with the result that, at short time spans ( $m=$ 50 ), even subclades from the right tail were somewhat influenced by the boundary and therefore very slightly skewed. Simulations using less abrupt (i.e., soft or graded) boundaries also gave results (not shown) qualitatively similar to those in figure 5 .

The suggestion has been made that the right skew of size distributions for many taxa could be the result of higher extinction probabilities at large size and/or higher branching probabilities 

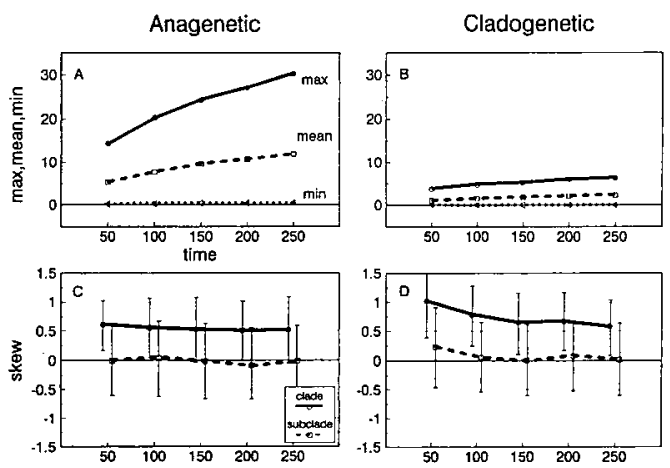

FIG. 5. Results of the model for a passive system. In this case, a sticky boundary (see text) is present at 0 . The graphs show the pattern of change in the parent clade maximum, mean, and minimum ( $A$ and $B$ ) and in the parent clade and subclade skew (C and D) for five time spans. Results are shown for both anagenetic (A and $\mathrm{C}$ ) and purely cladogenetic (B and D) systems. Points represent mean values for 200 successful runs. Error bars in C and D show one standard deviation. Here, and in subsequent graphs with error bars, points showing parent clade and subclade mean skews are offset somewhat from their proper abscissas so that error bars do not overlap.

at small size (Stanley 1973; Dial and Marzluff 1988; Brown and Nicoletto 1991). In some cases, the right skew may be an artifact of plotting on an arithmetic scale, but in others, the distribution remains skewed even on a log scale (Maurer et al. 1992 and references therein). Maurer et al. (1992) studied such branching and extinction heterogeneities, modeling them appropriately as changes in branching or extinction rate across a morphospace, and found that they do not tend to produce highly skewed distributions. In preliminary tests with the above model, similar results were obtained.

Maurer et al. (1992) also investigated an unbiased, unbounded system. They used a multiplicative model, found that it produced log-normal distributions, and concluded that a diffusive mechanism could not have produced the right skew in log-body-size distributions. The finding is important: if size distributions and distributions from this model were plotted on arithmetic scales, both would be skewed, and the erroneous conclusion might be drawn that an unbiased, unbounded model accounts for the shape of observed size distributions. Thus, the finding is a useful demonstration of the importance of choosing a proper scale. But it is not relevant to a passive (diffusive) mechanism, because the model contained no explicit boundary, which is a crucial element in all versions of the mecha-
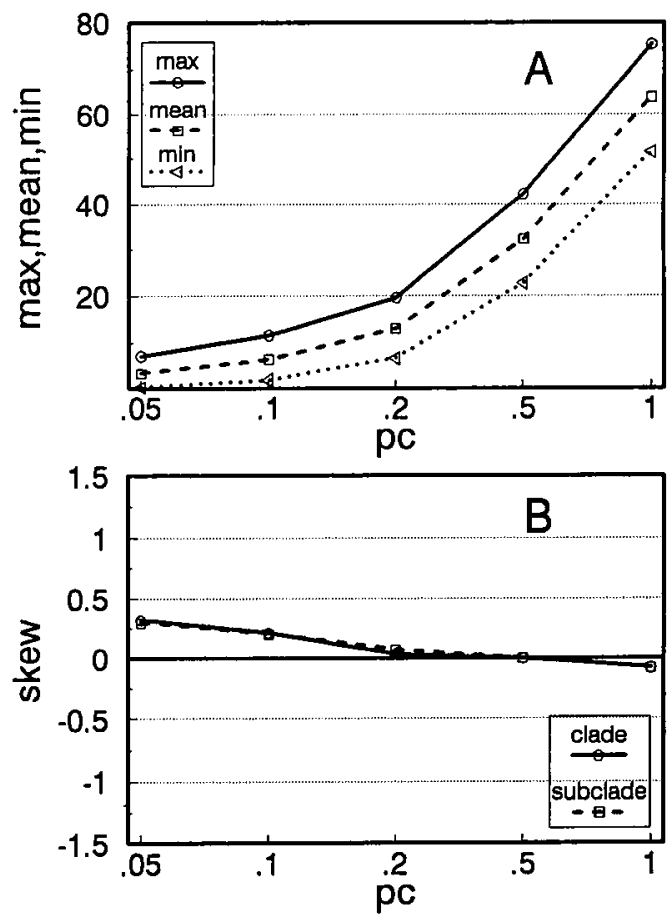

Fig. 6. Results of the model for a driven system (anagenetic), showing the parent clade maximum, mean, and minimum (A) and mean skew values (B) for parent clade and subclade as a function of the probability of change ( $p c=p c a$, see text). For all values of $p c$, the system had a strong bias: increases were nine times more probable than decreases. Note that the abscissa is not quite a homogeneous log scale; points are evenly spaced, but adjacent values do not have the same ratio in all cases.

nism (Fisher 1986; Gould 1988; McKinney 1990a).

\section{Driven Systems}

In a driven system, the space in which diversification occurs is homogeneous, that is, the direction or magnitude of change is biased to the same degree across the space. If the system is selection-driven, for example, and selection favors increases, then increases occur more frequently than decreases. In the model, a strong bias was introduced by making the probability of increase nine times greater than the probability of decrease, both in branching and in anagenesis. As shown in figure $6 \mathrm{~A}$, a strong bias produced dramatic increases in the mean and minimum, at least when the probability of change was high (e.g., $p c=1$ ), and the skew was near zero or even slightly negative.

However, notice that as the probability of change decreased, the increases in the mean and 

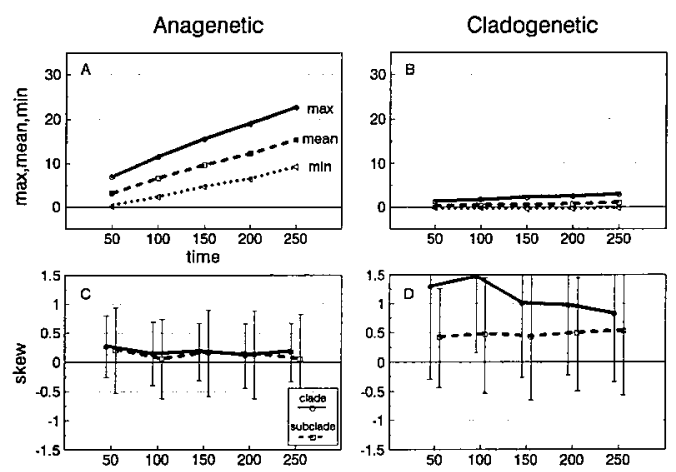

Fig. 7. Results of the model for a driven system. In this case, no boundary was present, but the system had a strong bias (increases nine times more likely than decreases), and the probability of change was low ( $p i$ $=p i a=0.09 ; p d=p d a=0.01 ; p c=p c a=0.10$ ). See the caption for figure 5 for further explanation.

minimum were reduced, and the skew for the parent clade increased (fig. 6B). At the lowest probability of change $(p c=0.05)$, the increase in the minimum was negligible and the parent clade skew was substantial, precisely the expectation for a passive system. However, the behavior of the subclades identifies this as a driven system. In the passive system, subclades had a mean skew close to zero (fig. 5C,D), but here the subclade mean skew was positive and close to the parent clade skew (fig. 6B).

Figure 7 shows the behavior over time of a driven system with a low probability of change $(p c=p c a=0.10)$. As time passed, the parent clade mean and minimum increased (fig. 7A,B). (Again, the increases were less dramatic in the cladogenetic system, because change was limited to speciation events.) At the shortest time span ( $m=50$ ), the skew of the driven system was positive and the minimum had hardly changed at all, effectively mimicking a passive system. Unlike the passive system, however, subclades in the driven system were somewhat positively skewed at all time spans (fig. 7C,D). (Results for a driven system in which the magnitude of change was biased, rather than the direction of change, were qualitatively the same.)

Note that in the anagenetic system, the minimum increased as time passed. (The minimum also increased in the cladogenetic system but very slowly, and imperceptibly in fig. 7D.) In effect, the system started to behave like the more familiar driven system in figure $1 \mathrm{~B}$, raising the possibility that a driven system with low probability of change can mimic a passive system only in the short run. Further exploration of the model's parameter space would be needed to confirm this, however.

\section{Distinguishing Passive ANd DRIVEn}

Three methods are available for distinguishing passive and driven systems. For all, the assumption is that a trend has either been established (or assumed on some reasonable grounds), with a trend understood to mean a consistent pattern of increase in the mean in a diversifying clade.

\section{The Minimum Test}

If the minimum increases, then the system is probably driven (fig. 7A). (It is worth noting again that the expected minimum for a passive system also increases, although only slightly [fig. 5A].) If the minimum decreases or remains constant, then the system may have originated to the right of, or right at, a boundary. As discussed, however, the system could instead be driven, and the decrease or stasis in the minimum could be transient. The analysis so far does not permit any quantitative estimate of how much time would correspond to long and short run in real systems. Thus, the minimum test is asymmetrical in that only one of the possible results, substantial increase in the minimum, has a single interpretation.

In principle, the test requires only two data points, an ancestral minimum and a terminal minimum, although the likelihood of increase or (near) stasis caused by chance alone would be high. The ideal data are paleontological time series showing consistent patterns of change (or stasis) in the minimum, such as those used by Gould (1988), or multiple comparisons (Jablonski 1987) so that patterns have the opportunity to achieve statistical significance. As for other morphological patterns, analyses should take into account expected changes resulting from rarefaction, which includes both decreases in diversity and differences in sample size even when diversity increases (Foote 1992).

\section{The Ancestor-Descendant Test}

In a passive system, increases are expected to occur as often as decreases, whereas in a driven system, increases should occur more often. The test requires a number of ancestor-descendant pairs lying far from any possible lower bound, that is, far from the clade minimum. This condition is important so that a boundary, if present, will not bias the outcome. If pairs in which an increase occurs significantly outnumber pairs that 
show a decrease, the system is probably driven. An equal number of increases and decreases, or a nonsignificantly greater number of increases, is consistent with either mechanism. However, if decreases outnumber increases, then the result is closer to the expected finding for a passive system (equal numbers of increases and decreases) than to the expected finding for a driven system (a greater number of increases), and the system is therefore more likely to be passive (assuming equal variances for mean skew values in both systems).

MacFadden (1986) used the ancestor-descendant test to study the well-known size trend in horses from the Eocene to the Pleistocene. In 24 ancestor-descendant pairs, he found 19 increases and 5 decreases. Among the pairs lying far from any possible lower bound-defined here as those in which either ancestor or descendant was larger than the mean log-size-increases outnumbered decreases 9 to 0 , a significant bias $(P<.002)$, which suggests a driven system.

The case is instructive. In figure 8 , the early size distribution is right skewed, consistent with either a passive or driven system. However, as time passes, the minimum increases, as expected if the system is driven. Thus, the minimum test and the ancestor-descendant test agree.

Boyajian has been using the ancestor-descendant test to study a Paleozoic and Mesozoic trend in mean suture complexity in ammonoids. Preliminary results are as follows: 5 increases and 6 decreases in 11 ancestor-descendant comparisons (Boyajian pers. comm. 1993). Results of the minimum test are equivocal. Minimum complexity increased somewhat for ammonoids as a whole in the diversification leading up to the endPermian mass extinction (Boyajian and Lutz 1992) but decreased somewhat in the diversification that followed. Also, the minimum decreased in a number of lower taxa (orders) (Boyajian pers. comm. 1993). If the decreases within orders are not chance or transient phenomena, then the system is passive, in agreement with the ancestor-descendant test. Actually, in this case, the system must be at least partly passive, because the earliest ammonoids had complexity values close to that for a straight line, the simplest possible suture.

The ancestor-descendant test is the most direct test of the three, but it requires a robust phylogeny that includes a substantial number of ancestors or near ancestors (relatively underived sister taxa). The limitation is serious, because reliable phylogenies are not available for most taxa, and
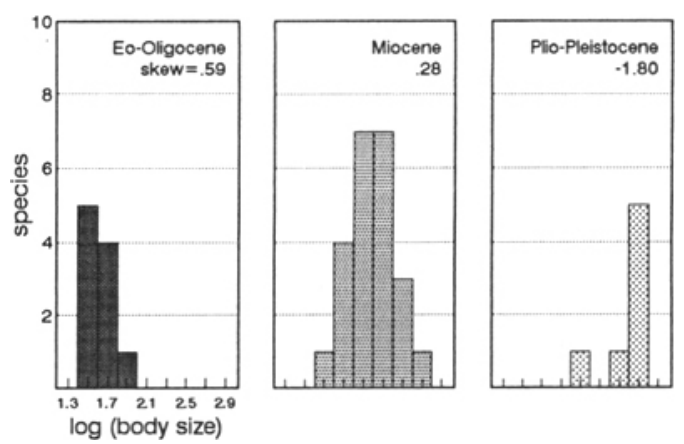

Fig. 8. A time series of log-body-size (kg) distributions for the Equidae (Mammalia, Perissodactyla). Data are from MacFadden (1986). Here, and in figures 9 and 10 , numbers on the abscissa indicate the upper limit of the corresponding category; for example, the first bar of the Miocene histogram indicates that there was one species in the 1.5-1.7 log-body-size category.

even good phylogenies may contain few or no ancestors.

\section{The Subclade Test}

If the parent distribution is skewed and the mean skew of a sample of subclades drawn from the tail is significantly positive, then the system is probably driven. A mean subclade skew that is positive but not significantly greater than 0 is consistent with either mechanism. But if the mean subclade skew is negative, then the system is more likely passive (again, assuming equal variances), because the negative mean is closer to the expectation for a passive system (zero) than to that for a driven system (positive).

In real cases, statistical tests to demonstrate the significance of skewness values are desirable. For the parent clade, however, this will sometimes be impossible. In single-occurrence trends, for example, we will have no distributions, only single skewness values. Standard tests for significance of single skewness values are not applicable, because they assume the members of the distribution are independent (Snedecor and Cochran 1980), and taxa originating in a branching process may be mutually constraining (Harvey and Pagel 1991). Subclades, however, if they are not nested, are truly independent. If a reasonable number of them can be found in the tail of the parent clade, a bootstrap of their mean skew can be used to test whether that mean is significantly different from zero.

For many trends, the parent distribution is not skewed, in which case, driven and passive cannot be reliably distinguished with this test. In par- 


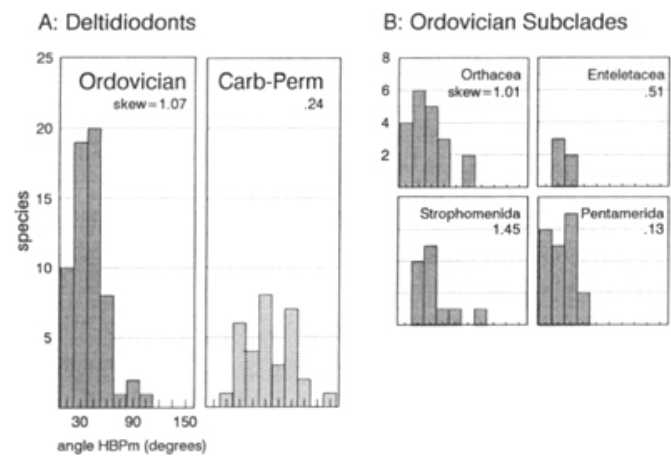

Fig. 9. A. An Ordovician distribution and a combined Carboniferous-Permian distribution for the hingediductor angle (angle HBPm; see text) in deltidiodont brachiopods. B. Distributions for four subclades drawn from the right tail of the Ordovician distribution. Data are from Carlson (1992). See the caption for figure 8 .

ticular, skewed subclades within an unskewed parent clade might suggest the presence of multiple boundaries in the tail region, or a local driven system in the tail, but provide no evidence for the cause of a larger-scale trend.

The subclade test requires only a terminal distribution so that, in principle, it ought to be possible to make inferences about evolutionary mechanisms using only a modern distribution of a state variable. However, the test assumes that the parameters of the system, such as branching and extinction rates, are stochastically constant over time, and paleontological data may be necessary to discover whether this assumption is likely to have been violated. For example, an intervening mass extinction would likely violate the assumption.

The assumption would also be violated in cases in which the terminal distribution is the result of a "filling in" of a preexisting distribution that is external to, and independent of, the system dynamic, such as the filling in of a preexisting distribution of niches in an ecosystem (if such occurs). At the point in time at which the external distribution is imposed, or at which its effect is first felt, the diversification dynamic becomes constrained, effectively altering the parameters of the system.

Finally, the ranges of parameters studied with the above model are a small subset of those possible for a diversifying system. Systems with extremely high diversity, long time spans, and diversity-dependent parameters were not considered, for example. Further investigation with the model is necessary to develop more confidence in the subclade test. In the meantime, the test should be used in conjunction with other tests when possible.

All three tests share the limitation that certain results are consistent with either mechanism (e.g., a static minimum, a nonsignificantly greater number of increases in a sample of ancestordescendant pairs, and a positive but nonsignificant mean subclade skew). Such results can still provide a certain amount of support for one mechanism or the other, however. If, for example, an increasing minimum suggests that a system is driven, then a mean subclade skew that is positive but not significant supports the driven mechanism to some extent. The nonsignificant result supports the driven interpretation by not contradicting it; in effect, the interpretation benefits by surviving a test.

\section{DEMONSTRATIONS}

\section{Brachiopod Geometry: A Driven System}

Carlson $(1989,1992)$ studied hinge and diductor-muscle geometry in Paleozoic brachiopods and found trends in a number of morphological variables. The left graph in figure 9A shows the distribution of values for Ordovician deltidiodont brachiopods in the angle formed by the hinge axis, the cardinal process, and the diductor muscle (angle HBPm in Carlson 1992, fig. 2). The distribution is positively skewed. The right graph, with the distribution for CarboniferousPermian deltidiodonts, shows that the mean and the minimum increased in the Paleozoic (Carlson 1992). Note that the Carboniferous-Permian distribution has 32 taxa, whereas the Ordovician has 61 , raising the possibility that the increase in the minimum is an artifact of rarefaction. However, that is unlikely in this case: assuming that the Ordovician distribution, with ten species in the lowest angle class, is representative of the underlying distribution, the probability that a sample of 32 taxa drawn from that distribution would have no representatives in the smallest class is 0.0032. (Carlson [1992, fig. 8] shows a more complete time series, and the minimum for all post-Ordovician time periods is greater than the Ordovician minimum.) Thus, the system was probably driven.

Figure 9B shows four subclades drawn from the tail of the Ordovician distribution; two have means greater than that of the original clade, and the other two are less but close (table 2). All four are positively skewed, suggesting that the system was driven. (The probability that four of four subclades would be positively skewed by chance in an unbiased system is 1 in 16 or 0.0625 .) 
TABLE 2. Sample sizes, means, and skew values.

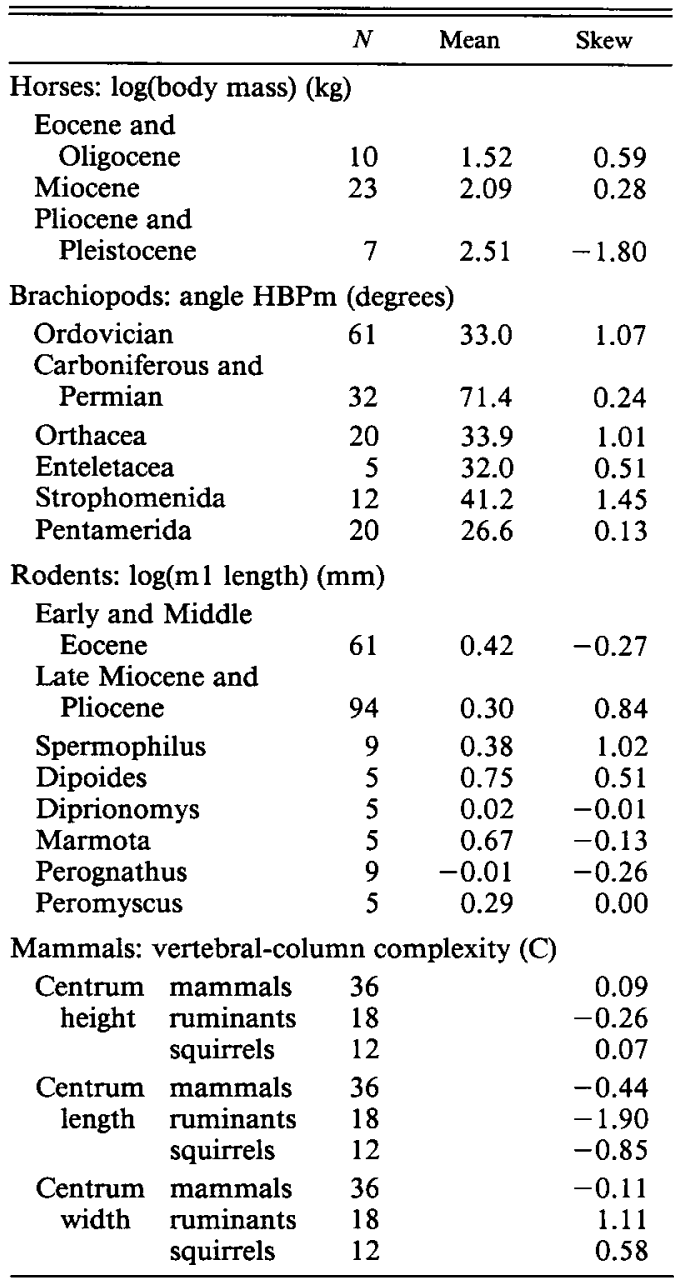

Finally, the possibility should be raised that the data are plotted on a biologically inappropriate scale. It is possible that change in brachiopod hinge-diductor geometry is nonlinear on the HBPm-angle scale, but linear on some other scale, and that the skew in both the large clade and the subclades is simply an artifact of the inappropriate scale. Further study of the developmental route to changes in geometry and function might help identify the proper scale. Meanwhile, it is worth noting that if the skews are artifacts of scale, the subclade test nevertheless accurately reports that the distortion of the morphospace is homogeneous, as expected from a scale problem. This is a useful reminder that the subclade test is a direct indicator of the homogeneity or heterogeneity of the space and only an indirect indicator of evolutionary process.

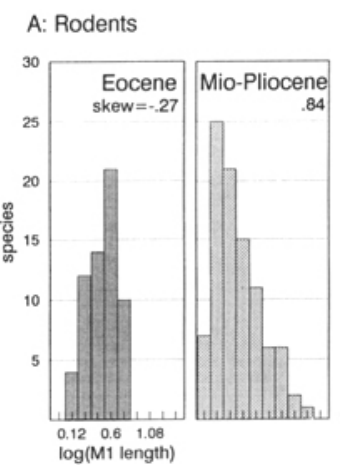

B: Mio-Pliocene Subclades

Fig. 10. A. A combined Early and Middle Eocene distribution (left) and a combined Late Miocene and Pliocene distribution (right) for log-lower-first-molar length $(\mathrm{mm})$ in North American rodents. B. Distributions for six subclades drawn from the Mio-Pliocene distribution. North American rodents are not a monophyletic group, but they are probably an unbiased sample of the worldwide rodent clade (Stanley 1973), which is adequate for present purposes. Data are from Stanley, supplemented with additional data from Hibbard (1939, 1941), Jacobs (1977), Lindsay (1972), Martin (1984), Repenning (1962), Shotwell (1970), and Zakrzewski $(1969,1984)$. See the caption for figure 8.

\section{Rodent Size: A Passive System}

Stanley (1973) presented data showing that distributions for anterior-posterior length of the first lower molar (which he uses as a size estimator) in North American rodents are right skewed (on a linear scale). I have added to Stanley's data, especially his data for North American rodents of the Late Miocene and the Pliocene (the Clarendonian, Hemphillian, and Blancan land-mammal ages, corresponding roughly to Stanley's "Pliocene"). Figure 10A shows distributions based on the enhanced data set for Eocene and Mio-Pliocene rodents. Plotted on a log scale, the Eocene distribution is left skewed, but the Mio-Pliocene is right skewed, indeed considerably so.

Stanley noted that minimum size decreased somewhat from the Eocene to the Pliocene, whereas mean size for the group as a whole increased (fig. 10A; see also a three-histogram time series in Stanley 1973, fig. 6). His interpretation was that the trend is (partly) accounted for by the presence of a lower bound on size.

Figure 10B shows distributions for six subclades drawn from the larger Mio-Pliocene distribution. Four of these, Spermophilus, Dipoides, Marmota, and Peromyscus, come from the tail. That is, their means are greater than (or very close to, in the case of Peromyscus) the parent clade mean (table 2). The mean skew for the four 
is 0.35 , and in a bootstrap test ( 1000 resamplings with replacement), this value was found to be significantly less than the parent clade skew of $0.84(P<.05)$, but not significantly greater than zero $(P<.05)$.

Thus, the minimum decreases, and the mean subclade skew is not significantly greater than zero. Both test results are consistent with a passive mechanism and thus support Stanley's conjecture to some extent.

\section{Complexity: A Passive System}

Vertebral columns of living and fossil fish are typically rather simple or undifferentiated, in that vertebral morphology changes little from one end of the column to the other. Mammalian columns are quite differentiated, however: cervical vertebrae differ considerably from thoracic vertebrae, which in turn differ from lumbars, and so on. Apparently, therefore, a trend in complexity of the vertebral column occurred at a high taxonomic level in the transition from fish to mammal (McShea 1993). The evolution of mammals certainly raised the maximum column complexity, and also probably the mean, in the vertebrate clade.

Further, even without morphometric data, it seems reasonable to suppose that the complexity distribution for vertebral columns is positively skewed. With about 28,000 fish species known, the greatest number of vertebrate species would seem to be concentrated at low column complexity values, with the 4000 more-complex mammal species adding a short tail on the right. Modern amphibian and reptile columns are fairly fishlike, and bird columns seem about as complex as mammal columns; thus, adding 9000 bird species and 10,000 amphibian and reptile species would not erase the right skew.

Test results indicate that the trend and skew are probably products of a passive system. First, an impressionistic survey of fossil fish vertebral columns (e.g., see Carroll 1988) suggests that minimum complexity has not changed much; the columns of modern fish are about as undifferentiated as those of Paleozoic fish.

For the ancestor-descendant test, quantitative measures of complexity were used. In earlier work (McShea 1992), I developed three metrics that capture complexity as functions of the amount of differentiation within a column (or any homologous series). For example, in one metric (C), the complexity of a column was measured as the $\log$ of the mean absolute difference, in a single vertebral dimension, between each vertebra and the mean for the column. (For further discussion of the metrics and size correction methods, see McShea 1992, 1993.) The metrics were applied in a random sample of ancestor-descendant comparisons within mammals. Summing over all three metrics, 9 significant increases and $13 \mathrm{sig}$ nificant decreases occurred (McShea 1993), consistent with an unbiased system.

For the subclade test, skew values were computed for samples from three vertebrate subclades in three vertebral dimensions (table 2). The larger subclade consisted of 36 mammal specimens, each belonging to a different mammal family. The smaller subclades were the ruminants (represented by 18 specimens in 18 different genera) and the sciurids (represented by 12 specimens in 12 genera). The two smaller subclades (ruminants and squirrels) are nested within the third (mammals); thus, they are not strictly speaking independent of it. However, the difference in taxonomic level is so large that the constraint is probably weak.

The mean skew for two individual dimensions (centrum height and length) is negative, all three dimensions contain at least one negative value, and the grand mean for all nine skew values is negative ( -.19$)$, all consistent with a passive system.

In addition to these empirical results, one line of reasoning supports the existence of a lower bound. The vertebrae of a typical fish column are very similar to each other; thus, fish columns are about as undifferentiated as possible. Thus, it seems inescapable that a lower bound, defined analytically (as in the ammonoid case), exists at complexity values very near those of fish columns.

\section{CONCLUSION}

In summary, large-scale trends may be passive, resulting from diffusion in bounded spaces, or driven, resulting from pervasive biases in the direction of change among lineages. The distinction is not relevant to such salient issues in evolutionary biology as whether trends are caused by internal or external factors, by selection or developmental constraints, because both boundaries and biases may be caused by either. Rather, it concerns the distribution of constraints or selective forces in the state space. In a passive trend, the distribution is heterogeneous, with constraints or forces acting only at a boundary, whereas in a driven system, the distribution is homogeneous, with either factor or both acting throughout the space. Like homogeneity and het- 
erogeneity, passive and driven identify the extremes on a single continuum, and they are scale relative.

The significance of the passive-driven distinction lies in the independence among hierarchical levels implied by the passive mechanism, a counterintuitive notion for many. If this independence is overlooked, it is easy to think that largescale behavior must be nothing but small-scale behavior amplified and to make unjustified inferences from small to large scale. The reverse inference is equally facile. For example, Jastrow (1981) discovered a trend in primates toward greater intelligence and inferred that, if past forces continue to operate, intelligence is likely to increase in the human lineage. If such a trend in primates exists and if it is driven, that is, if the trend is a direct result of concerted forces acting on most lineages across the intelligence spectrum, then the inference is justified. But if it is passive, that is, if forces act only on lineages at the low-intelligence end, then most lineages will have no increasing tendency. In that case, most primate species-especially those out on the right tail of the distribution like ours-would be just as likely to lose intelligence as to gain it in subsequent evolution (if they change at all). More generally, inference from large to small scale, or vice versa, is warranted for driven systems but not for passive. Thus, to justify such inferences, whether they are used in prediction or, as is more common, retrodiction (historical analysis), knowledge of the trend mechanism is essential.

The modeling and the demonstrations illustrate some of the tests that can be used to distinguish passive from driven systems: (1) the test based on the behavior of the minimum; (2) the ancestor-descendant test, based on numbers of increases and decreases in random samples of ancestor-descendant pairs that lie far from any possible boundary; and (3) the subclade test, based on the mean skew of subclades drawn from the right tail of the terminal distribution. A system is probably driven if the minimum increases, if increases significantly outnumber decreases among ancestor-descendant pairs, and if the mean skew of subclades is significantly positive. A passive mechanism is more difficult to establish, but is the more likely mechanism if decreases outnumber increases and if the mean skew of subclades is negative.

The minimum test requires a paleontological time series of distributions (to reliably establish the behavior of the minimum), and the ancestordescendant test requires a robust phylogeny con- taining many ancestral or near-ancestral taxa. The subclade test has the advantage that-assuming a trend has been established or can reasonably be assumed -it requires no trend history at all, just a single skewed terminal distribution. In principle, then, a modern distribution can provide evidence for the mechanism behind a largescale trend, and no paleontological data are needed. However, it is worth noting again that the model has been used to explore only a small region of parameter space, and the behavior of the skew in other regions is unknown. At least at present, therefore, the subclade test should be supplemented with other tests.

One other caveat deserves equal billing. The tests assume that the parameters of the system are stochastically constant in time. In cases in which there is reason to think the assumption of constancy is violated, the tests should not be applied.

In the trends examined, the various tests gave concordant results. In the horses, minimum size increased from the Eocene to the Pleistocene and the ancestor-descendant test indicated a branching bias, both consistent with a driven system. In ammonoids, the results were somewhat equivocal: minimum suture complexity increased slightly in the group as a whole, but the decreases in minima within orders and the absence of a branching bias are both consistent with a passive system. Further, the existence of a lower bound can be inferred analytically.

In brachiopods, the post-Ordovician minimum hinge-diductor angle increased and the subclades were skewed, both consistent with a driven system. In rodents, the minimum decreased, and the mean subclade skew was positive but not significantly different from zero, both consistent with passive system. Finally, the minimum complexity of vertebral columns probably did not change (indeed, the actual minimum seems to have remained close to the theoretical minimum), ancestor-descendant comparisons in subclades of mammals revealed no branching bias, and the mean subclade skew was negative, all pointing to a passive system.

The forces at work in the diversification of large-scale trends are probably complex, as discussed, and it is therefore surprising that the test results concur. Indeed, when more systems have been investigated, these results may turn out to be atypical. At present, however, given the concurrence, it is tempting to speculate that results for skewed clades may commonly fall into two discrete syndromes: the driven syndrome, de- 
fined by an increasing minimum, biased branching, and skewed subclades; and the passive syndrome, defined by a stable or decreasing minimum, unbiased branching, and unskewed subclades. At least, these results do raise the possibility that the passive and driven mechanisms may be natural categories and that they may correspond to distinct and well-defined causes of large-scale trends.

\section{ACKNOWLEDGMENTS}

Thanks to M. Foote for many useful discussions. For thoughtful reviews of the manuscript, I thank C. Badgley, D. Fisher, M. Foote, N. Gilinsky, P. Gingerich, M. LaBarbera, B. Maurer, M. McKinney, D. Raup, D. Ritchie, L. Van Valen, and G. Vermeij. For data, I thank S. Carlson, S. Stanley, G. Boyajian, D. Norris, and B. MacFadden. B. Miljour and D. Ritchie kindly helped with some of the figures. This research was supported by the Michigan Society of Fellows.

\section{Literature Cited}

Alberch, P. 1980. Ontogenesis and morphological diversification. American Zoologist 20:653-667.

Berg, H. C. 1993. Random walks in biology. Princeton University Press, Princeton, N.J.

Bonner, J. T. 1988. The evolution of complexity. Princeton University Press, Princeton, N.J.

Bottjer, D. J., and D. Jablonski. 1988. Paleoenvironmental patterns in the evolution of post-Paleozoic benthic marine invertebrates. Palaios 3:540-560.

Boyajian, G., and T. Lutz. 1992. Evolution of biological complexity and its relation to taxonomic longevity in the Ammonoidea. Geology 20:983986.

Brooks, D. R., and E. O. Wiley. 1988. Evolution as entropy, 2d ed. University of Chicago Press, Chicago.

Brown, J. H., and P. F. Nicoletto. 1991. Spatial scaling of species composition: Body masses of North American land mammals. American Naturalist 138: 1478-1512.

Carlson, S. J. 1989. The articulate brachiopod hinge mechanism: Morphological and functional variation. Paleobiology 15:364-386.

- 1992. Evolutionary trends in the articulate brachiopod hinge mechanism. Paleobiology 18:344 366.

Carroll, R. L. 1988. Vertebrate paleontology and evolution. W. H. Freeman, New York.

Cope, E. D. 1871. The method of creation of organic forms. Proceedings of the American Philosophical Society 12:229-263.

Damuth, J. 1993. Cope's rule, the island rule and the scaling of mammalian population density. Nature 365:748-750.

Dial, K. P., and J. M. Marzluff. 1988. Are the smallest organisms the most diverse? Ecology 69:1620-1624.
Fisher, D. C. 1986. Progress in organismal design. Pp. 99-117 in D. M. Raup and D. Jablonski, eds. Patterns and processes in the history of life. Springer, Berlin.

Foote, M. 1992. Rarefaction analysis of morphological and taxonomic diversity. Paleobiology 18:116.

Gould, S. J. 1988. Trends as changes in variance: a new slant on progress and directionality in evolution. Journal of Paleontology 62:319-329.

- 1990. Speciation and sorting as the source of evolutionary trends, or "things are seldom what they seem." Pp. 3-27 in K. J. MacNamara, ed. Evolutionary trends. University of Arizona Press, Tucson.

Harvey, P. H., and M. D. Pagel. 1991. The comparative method in evolutionary biology. Oxford University Press, Oxford.

Hibbard, C. W. 1939. Notes on additional fauna of Edson Quarry of the Middle Pliocene of Kansas. Transactions of the Kansas Academy of Science 42: 457-462.

- 1941. New mammals from the Rexroad Fauna, Upper Pliocene of Kansas. American Midland Naturalist 26:337-368.

Jablonski, D. 1987. How pervasive is Cope's rule? A test using Late Cretaceous mollusks. Geological Society of America. Abstracts 19:713-714.

Jablonski, D., and D. J. Bottjer. 1990. Onshore-offshore trends in marine invertebrate evolution. $\mathrm{Pp}$. 21-75 in R. M. Ross and W. D. Allmon, eds. Causes of evolution. University of Chicago Press, Chicago.

Jacobs, L. L. 1977. Rodents of the Hemphillian Age Redington local fauna, San Pedro Valley, Arizona. Journal of Paleontology 51:505-519.

Jastrow, R. 1981. The enchanted loom. Simon and Schuster, New York.

LaBarbera, M. 1986. The evolution and ecology of body size. Pp. 69-98 in D. M. Raup and D. Jablonski, eds. Patterns and processes in the history of life. Springer, Berlin.

Lindsay, E. H. 1972. Small mammal fossils from the Barstow Formation, California. University of California Publications in Geological Science 93:1-104.

MacFadden, B. J. 1986. Fossil horses from "Eohippus" (Hyracotherium) to Equus: Scaling, Cope's law, and the evolution of body size. Paleobiology 12 : 355-369.

Martin, J. E. 1984. A survey of Tertiary species of Perognathus (Perognathinae) and a description of a new genus of Heteromyinae. Pp. 90-121 in R. M. Mengel, ed. Papers in vertebrate paleontology honoring Robert Warren Wilson. Special Publication of the Carnegie Museum of Natural History, 9.

Maurer, B. A., J. H. Brown, and R. D. Rusler. 1992. The micro and macro in body size evolution. Evolution 46:939-953.

Maynard Smith, J. 1970. Time in the evolutionary process. Studium Generale 23:266-272.

McKinney, M. L. 1990a. Classifying and analysing evolutionary trends. Pp. 28-58 in K. J. MacNamara, ed. Evolutionary trends. University of Arizona Press, Tucson.

75. 1990b. Trends in body-size evolution. Pp. 75-118 in K. J. MacNamara, ed. Evolutionary trends. University of Arizona Press, Tucson. 
McShea, D.W. 1991. Complexity and evolution: What everybody knows. Biology and Philosophy 6:303324.

- 1992. A metric for the study of evolutionary trends in the complexity of serial structures. Biological Journal of the Linnean Society 45:39-55.

- 1993. Evolutionary change in the morphological complexity of the mammalian vertebral column. Evolution 47:730-740.

Newell, N. D. 1949. Phyletic size increase, an important trend illustrated by fossil invertebrates. Evolution 3:103-124.

Raup, D. M. 1977. Stochastic models in evolutionary paleontology. Pp. 59-78 in A. Hallam, ed. Patterns of evolution. Elsevier, Amsterdam.

1985. Mathematical models of cladogenesis. Paleobiology 11:42-52.

. 1988. Testing the fossil record for evolutionary progress. Pp. 293-317 in M. Nitecki, ed. Evolutionary progress. University of Chicago Press, Chicago.

- 1991. A kill curve for Phanerozoic marine species. Paleobiology 17:37-48.

Raup, D. M., and S. J. Gould. 1974. Stochastic simulation and evolution of morphology - towards a nomothetic paleontology. Systematic Zoology 23: 305-322.

Repenning, C. A. 1962. The giant ground squirrel Paenemarmota. Journal of Paleontology 36:540 556.

Salthe, S. N. 1993. Development and evolution. MIT Press, Cambridge.

Saunders, P. T., and M.-W.Ho. 1976. On the increase in complexity in evolution. Journal of Theoretical Biology 63:375-384.

Sepkoski, J. J. 1991. A model of onshore-offshore change in faunal diversity. Paleobiology 17:58-77.
Shotwell, J. A. 1970. Pliocene mammals of Southeast Oregon and adjacent Idaho. Museum of Natural History, University of Oregon, Bulletin 17.

Skellam, J. G. 1951. Random dispersal in theoretical populations. Biometrika 38:196-218.

Slatkin, M. 1981. A diffusion model of species selection. Paleobiology 7:421-425.

Snedecor, G. W., and W. G. Cochran. 1980. Statistical methods, 7 th ed. Iowa State University, Ames.

Sober, E. 1984. The nature of selection. MIT Press, Cambridge, Mass.

Stanley, S. M. 1973. An explanation for Cope's rule. Evolution 27:1-26.

. 1979. Macroevolution. W. H. Freeman, San Francisco.

Toft, C. A., and M. Mangel. 1991. Discussion: From individuals to ecosystems; the papers of Skellam, Lindeman and Hutchinson. Bulletin of Mathematical Biology 53:121-134.

Van Valen, L. 1973. A new evolutionary law. Evolutionary Theory 1:1-18.

Vermeij, G. J. 1987. Evolution and escalation. Princeton University Press, Princeton.

Wicken, J.S. 1987. Evolution, thermodynamics, and information. Oxford University Press, Oxford.

Zakrzewski, R. J. 1969. The rodents from the Hagerman local fauna, Upper Pliocene of Idaho. Contributions from the Museum of Paleontology, The University of Michigan 23:1-36.

1984. New arvicolines (Mammalia, Rodentia) from the Blancan of Kansas and Nebraska. Special Publications of the Carnegie Museum 8:200217. 